Images dans le monde ibérique et ibéricoaméricain

9 | 2016

Innovations politiques et culturelles dans les pays andins

\title{
Les évolutions de la législation cinématographique dans les pays andins
}

Reflet d'un changement sociétal

\section{Christine Delfour}

\section{(2) OpenEdition \\ Journals}

\section{Édition électronique}

URL : http://journals.openedition.org/agedor/1192

DOI : 10.4000/agedor.1192

ISSN : 2104-3353

\section{Éditeur}

Laboratoire LISAA

\section{Référence électronique}

Christine Delfour, "Les évolutions de la législation cinématographique dans les pays andins », LÂAge d'or [En ligne], 9 | 2016, mis en ligne le 01 mars 2016, consulté le 19 avril 2019. URL : http:// journals.openedition.org/agedor/1192; DOI : 10.4000/agedor.1192

Ce document a été généré automatiquement le 19 avril 2019.

L'Âge d'or. Images dans le monde ibérique et ibéricoaméricain 


\title{
Les évolutions de la législation cinématographique dans les pays andins
}

\author{
Reflet d'un changement sociétal
}

\section{Christine Delfour}

1 Le retour à la démocratie dans la plupart des pays latino-américains dans les deux dernières décennies $\mathrm{du} \mathrm{XX}^{\mathrm{e}}$ siècle marque un tournant dans le monde de la culture et dans une de ses expressions emblématiques: le cinéma. Démocratie et manifestations culturelles sont étroitement liées. Ces manifestations façonnent les imaginaires nationaux même si, aujourd'hui, on ne peut plus parler de cinéma engagé mais plutôt de cinéma militant. Ce phénomène est d'autant plus affirmé qu'il correspond, dans la plupart des pays andins (Bolivie, Colombie, Équateur, Pérou et Venezuela), à des changements de sociétés ${ }^{1}$. On assiste, à partir de la victoire de Chávez au Venezuela, en 1998, à des élections qui portent régulièrement au pouvoir dans cette première décennie du XXI ${ }^{e}$ siècle, des gouvernements de gauche ou de centre-gauche (le Brésil avec Lula, l'Argentine avec Nestor Kirchner, l'Uruguay avec le Frente Amplio, la Bolivie avec Evo Morales, Correa en Equateur, etc.). Il s'agit d'une rupture réelle avec le passé: reconnaissance constitutionnelle de l'interculturalité et de la pluri-nationalité, frein à l'invisibilité sociale et politique des populations indiennes, contrôle par les États des ressources naturelles (nationalisations), politiques publiques volontaristes, etc. Le militantisme cinématographique passe, par exemple en Bolivie, par l'implication de cinéastes (Marcos Loyaza ou Juan Carlos Valdivia) dans des productions, commandes de l'État plurinational ou du Programme des Nations Unies pour le développement (PNUD) avec un objectif " pédagogique »: El Estado de las cosas (2007) et Laberinto (2007) ${ }^{2}$.

2 Ces changements sociétaux coïncident aussi avec le passage du celluloïd au numérique. Les pays andins n'y échappent pas, bien au contraire. La vidéo est devenue le principal media alternatif d'expression audiovisuelle. Elle démultiplie les créations dans tous les genres cinématographiques de plus ou moins bonne qualité. Demandant peu d'investissement, informel (pratiquement pas encore encadré par l'État), il est au cinéma 
ce que l'économie informelle est à l'économie formelle. Il est aussi le média avec lequel se sont formés de nombreux cinéastes reconnus ${ }^{3}$. D'autres faits participent à la généralisation de la vidéo: prolifération du DVD, des vidéoclubs, des chaînes de télévisions privées $^{4}$, disparition progressive des salles commerciales et réduction des

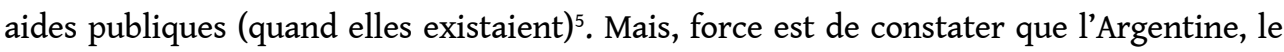
Brésil, et le Mexique sont aujourd'hui encore les pays qui produisent la plus grande quantité de films ${ }^{6}$, du fait d'une législation adaptée, de moyens importants alloués et d'une politique publique de diffusion et d'internationalisation de leurs industries cinématographiques.

3 Existe-t-il au niveau continental une volonté des États de protéger la création cinématographique? Les législations évoluent-elles en Bolivie, en Colombie, en Équateur, au Pérou au rythme soutenu des productions numériques? Quelles mesures, si elles existent, sont prises institutionnellement pour développer, financer et protéger la création cinématographique ? Tel est l'objet de notre réflexion.

\section{Des initiatives continentales}

4 L'« Accord pour la création d'un marché commun cinématographique latino-américain » ${ }^{7}$ (novembre 1989), renommé «Accord Ibéro-américain de coproduction cinématographique» (juillet 2006) et le programme Ibermedia (1998) sont les deux initiatives essentielles dans le domaine de la cinématographie latino-américaine ${ }^{8}$. Dans le premier cas, l'accord exprime la volonté des États signataires « de la nécessité d'impulser le développement cinématographique et audiovisuel de la région et, tout spécialement, dans les pays qui sont dotés d'une infrastructure insuffisante $»^{9}$. Il postule aussi pour que « l'activité cinématographique [...] contribue au développement culturel de la région et à son identité $\aleph^{10}$. Cet accord est signé seulement par 10 États ${ }^{11}$. En 2006, l'Espagne et la Colombie deviendront des membres à part entière de cet accord même si l'implication espagnole est effective depuis la mise en place du Programme Ibermedia en 1998. L'objectif est de créer, d'une part, un système multilatéral de création et de protection de productions cinématographiques certifiées « nationales » et, d'autre part, de développer un marché du cinéma des peuples ibéro-américains et des Caraïbes ${ }^{12}$.

5 Chacun des États membres s'engage à participer annuellement au Marché Commun avec, au moins, 4 films nationaux d'une durée de plus de 70 minutes. Des co-productions peuvent être envisagées entre États membres à partir d'accords bilatéraux entre les pays concernés qui viennent s'ajouter à l'offre annuelle du Marché. Ces co-productions sont aussi considérées comme nationales par les autorités compétentes de chaque État partenaire et bénéficient de plein droit des avantages fiscaux des pays membres. La participation financière de chacun varie de $20 \%$ à $80 \%$ par production ${ }^{13}$. Des coproductions multilatérales sont désormais possibles (2000). Un ou des co-producteurs peuvent artistiquement ou techniquement y participer de même qu'un autre ou que d'autres ont la possibilité de le faire seulement avec des capitaux. La participation de ce dernier ne peut être inférieure à $10 \%$ ni supérieure à $25 \%$. Les pays membres, quant à eux, doivent réaliser un apport d'au moins 10 \% supérieur au coût total de la production et ne peuvent excéder $70 \%{ }^{14}$.

6 Les pays signataires ont ensuite constitué la CACI (Conférence des autorités cinématographiques ibéro-américaines) et se sont engagés à participer à « l'intégration desdits pays au moyen d'une participation équitable à l'activité cinématographique 
régionale $»^{15}$. Enfin, il s'agit d'harmoniser les politiques des parties concernées en matière de cinéma et d'audiovisuel et de résoudre les problèmes de production, de distribution et de diffusion de la cinématographie de la région. Cette initiative a permis la diffusion de films latino-américains dans des salles nationales même si le public n'est pas toujours au rendez-vous, sollicité par les grandes productions nord-américaines distribuées dans la majorité des salles, et, d'autre part, elle a contraint les institutions des pays partenaires à instaurer des mesures en faveur du cinéma et de l'audiovisuel en général.

7 Le Programme Ibermedia, intégré par l'Espagne et le Portugal et la majorité des pays latino-américains, est l'initiative la plus remarquée ${ }^{16}$ et celle qui, à ce jour, fonctionne le mieux. Elle a été mise en œuvre officiellement au Sommet des chefs d'État ibéroaméricains, au Venezuela en 1998 en tant que fonds d'aide à la cinématographie latinoaméricaine. Au cours de ses dix premières années d'existence (1998-2008), Ibermedia a accordé près de 1200 aides au développement sous différentes formes, pour un total d'environ 58 millions de dollars. Ces fonds proviennent des apports réguliers que s'engagent à verser les différents organismes gouvernementaux responsables du cinéma lorsqu'ils intègrent le programme. Ibermedia représente actuellement une vingtaine de pays de la région et développe plusieurs lignes d'action : la coproduction (environ $80 \%$ des ressources), le développement de projets (entre $8 \%$ et $10 \%$ ), la distribution et la promotion (presque $6 \%$ ) ainsi que la formation (entre $4 \%$ et $5 \%)^{17}$. Selon les dernières évaluations (2015), Ibermedia a soutenu 636 projets de co-productions ibéro-américaines, 698 projets audiovisuels, tout en contribuant à l'emploi de 1200 entreprises et plus de 6000 professionnels. Il a participé à la promotion et distribution de 270 films. Plus de 2700 bourses de formation ont été réparties dans 19 pays. Enfin, les productions nationales latino-américaines sont désormais présentes dans les principaux festivals internationaux, de Berlin à Rotterdam, de Cannes à La Havane, de Los Angeles à Mar del Plata, de Huelva à Sundance ou de Toronto à San Sebastián. Certains films ont été nommés pour les Oscar à Hollywood $^{18}$.

8 Ce sont les pays à faible production et les petites entreprises cinématographiques qui sont les grands bénéficiaires du programme Ibermedia. Mais Octavio Getino remarque avec justesse que :

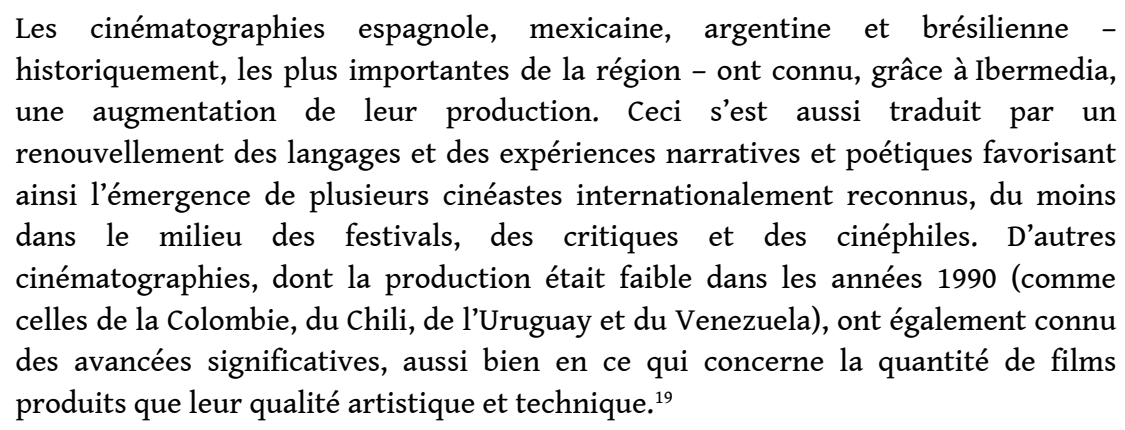

9 L'impact a peut-être été moindre au Pérou, en Bolivie ou en Équateur, mais néanmoins substantiel pour la survie de la création cinématographique face aux politiques inexistantes, insuffisantes ou erratiques des institutions de ces pays.

\section{Les législations dans les pays andins}

Un rapide panorama des législations en matière de cinéma dans ces pays révèle un empressement à légiférer et un immobilisme à appliquer : Loi 1302 (décembre 1991) en 
Bolivie ; Loi 814 de développement de l'activité cinématographique en Colombie (juillet 2003 ${ }^{20}$ ), Loi 2006-29 en Équateur ${ }^{21}$, Loi du cinéma (mars 1972) puis Loi 26370 (octobre 1994) au Pérou mais des lois obsolètes pratiquement pas appliquées où le faible soutien financier de l'État est évident. Certes la législation dans le domaine des arts et de la culture est nécessaire pour que la société puisse développer les manifestations culturelles d'une nation. Encore faut-il, d'une part, les appliquer sur le temps long, c'est-à-dire audelà des changements de gouvernements et, d'autre part, libérer des fonds conséquents pour la création, production, distribution et protection des œuvres. Nous nous attarderons sur les législations péruvienne et bolivienne.

11 La loi du cinéma de 1994, au Pérou, s'est révélée, dès sa publication, inopérante. L'État, sous prétexte de priorités gouvernementales, a réduit le montant annuel du budget dédié au cinéma, soit 2 millions de dollars annuels, au cinquième de la somme totale allouée ${ }^{22}$. Les sommes débloquées l'ont été à contretemps, sous forme de versements irréguliers. Les conséquences ont été dramatiques pour les producteurs et les réalisateurs, même si la généralisation numérique a permis de réduire les coûts de production et d'augmenter le nombre de productions (de plus ou moins bonne qualité) ${ }^{23}$. D'autant plus que la loi se limite à soutenir, par l'organisation de concours, la production de créations cinématographiques nationales (fondamentalement à Lima).

Les cinéastes se mobilisent et présentent, aujourd'hui, un avant-projet de réforme à contre-courant de la loi $\mathrm{N}^{\circ} 26370$, dont l'objectif est « d'aborder de manière intégrale le développement de l'activité cinématographique et audiovisuelle péruvienne (...) en associant tant la formation que les étapes de la création, de la production et de la commercialisation, ainsi que la préservation des œuvres $\aleph^{24}$. Il s'agit, d'une part, de multiplier une production cinématographique de meilleure qualité tout en variant les formats et les genres et, d'autre part, de former les publics et d'offrir de meilleures qualifications et spécialisations aux professionnels. Il s'avère nécessaire, enfin, de mettre en place une politique de communication des activités cinématographiques (publications de revues spécialisées et de divulgation) tant dans le pays qu'à l'extérieur.

Cette initiative législative, si elle devient réalité, peut s'effectuer sans coût additionnel pour l'État en réorientant les fonds perçus au nom de l'impôt sur les spectacles cinématographiques ${ }^{25}$ vers le Fonds pour le développement de la cinématographie et l'audiovisuel péruvien ${ }^{26}$. Cet avant-projet émet des propositions également en termes de défiscalisation pour les entreprises et les individus, investisseurs potentiels dans la production cinématographique. Dans ce nouveau cadre, la multiplication des ressources et l'augmentation de l'offre de films et de productions audiovisuelles entraînent la création d'emplois nouveaux et d'activités annexes (sécurité, alimentation, maintenance, etc.).

Indiquons cependant que, dans l'attente de la modification de la Loi $\mathrm{N}^{\circ} 26370$, les cinéastes et producteurs péruviens ont fait appel au Programme Ibermedia, au Fondo Iberamericano de Ayuda impulsé par l'Espagne et à différents fonds cinématographiques européens pour pallier les déficiences institutionnelles. Ce sont surtout des coproductions présentées, avec succès, dans des festivals internationaux. Le développement de la vidéo analogique puis l'irruption des supports numériques dans les années 1990 et 2000 ont multiplié les productions de courts métrages mais aussi de longs métrages à très petit budget, parfois de mauvaise qualité, avec des acteurs non professionnels. 
législation (1991) mal financée et peu appliquée. Et pourtant, elle a été discutée pendant une dizaine d'années par des cinéastes et réalisateurs importants sous le slogan : « Avoir un cinéma à nous, une image à nous " $^{27}$. L'absence de valorisation du cinéma par l'État a retardé la promulgation de la loi de financement de 1991 et a freiné les opportunités de faire un cinéma national de meilleure qualité. Toutefois, signalons deux initiatives fondamentales dans le cadre de cette loi : la création du Conseil National du Cinéma (CONACINE) qui doit « impulser, développer, coordonner, encadrer et mettre en place les activités cinématographiques dans le cadre de ses compétences, pour le développement du cinéma bolivien ${ }^{28}$ et un Fonds de développement à la production cinématographique nationale (art.15) ${ }^{29}$. Soulignons la participation dans la gestion publique de CONACINE de membres de la société civile avec l'État. Une vraie révolution dans la culture institutionnelle bolivienne du XXe siècle. Enfin, le chapitre VI de la loi est consacré au développement de la culture cinématographique en partenariat avec le Ministère de l'Éducation et de la Culture. Il s'agit fondamentalement d'un diplôme, niveau licence, pour la formation de cinéastes et techniciens audiovisuels dans les universités du pays ${ }^{30}$. Mais encore faut-il prévoir les débouchés professionnels pour ces futurs diplômés si la filière cinématographique n'est pas financée !

Malgré l'existence d'une législation dédiée au cinéma, il est vrai que les institutions et le public ignorent, en Bolivie, que « le cinéma n'est pas uniquement une activité artistique mais aussi une activité économique et sociale » comme le souligne Gerardo Guerra Velazco $^{31}$. Celle-ci est la pierre d'achoppement de la loi de 1991 et de ses limites en matière de Fonds de développement. La mobilisation a été très importante dès 2004, pour aboutir à une évaluation de la loi de 1991 impulsée par le directoire de CONACINE en 2011. La scène politique nationale s'est transformée radicalement dès 2005, avec la victoire d'Evo Morales aux élections nationales et avec la présence hégémonique de son parti, le Mouvement vers le socialisme (MAS), au sein de toutes les institutions. La mobilisation s'est faite plus forte. De nouveaux collectifs et de nouveaux cinéastes se sont mis en marche sur tout le territoire national ${ }^{32}$ pour débattre et rédiger collectivement la nouvelle loi du cinéma ${ }^{33}$ de la manière la plus démocratique et la plus participative possible dans l'esprit de la Nouvelle Constitution Politique de l'État plurinational ${ }^{34}$. Ils considèrent que :

La meilleure, la forme constitutionnelle, éthiquement et politiquement correcte pour faire des lois, c'est la participation citoyenne démocratique, plurielle, active, informée, suffisante et bienveillante. Une loi qui doit régir nos destins dans les prochaines décennies, sera bien meilleure, plus efficace et démocratique si nous l'élaborons entre nous tous qui en avons besoin pour développer notre secteur, entre nous tous qui en connaissons les forces et les faiblesses ainsi que les défis auxquels nous sommes confrontés, les objectifs et les horizons que nous désirons atteindre. ${ }^{35}$

17 Force est de constater qu'il n'existe pas dans ce pays une tradition narrative cinématographique nationale et que le public a abandonné le cinéma national pour les productions nord-américaines des cine centers ${ }^{36}$. Le public bolivien se méfie des productions nationales d'autant plus qu'elles sont souvent de mauvaise qualité. Il existe un fossé entre les cinéastes d'avant-garde ${ }^{37}$ qui expérimentent avec le cinéma un langage nouveau et qui n'ont pas ou peu accès aux salles traditionnelles (paradoxalement, ils ont accès aux salles de cinéma d'art et d'essai à l'étranger ${ }^{38}$ et et ceux qui font un cinéma 
médiocre, presque amateur, qui envahit les salles et qui se veut le représentant du cinéma bolivien pour le grand public ${ }^{39}$.

Force est de constater également que, malgré l'enthousiasme incarné par l'arrivée au pouvoir du « Président des mouvements sociaux » tel qu'aime se définir Evo Morales et que, malgré la mobilisation ${ }^{40}$ et les propositions des acteurs du monde cinématographique et des médias, la législation de 1991 est toujours en vigueur, depuis fort longtemps inapplicable, inutile et obsolète. L'État considère que la priorité est aujourd'hui à la mise en place de la Loi cadre des Cultures qui doit réunir tous les secteurs de la culture dont le cinéma. L'État reconnaît néanmoins que le caractère spécifique et singulier du cinéma doit être pris en compte. Une norme particulière est envisagée mais elle doit faire partie intégrante de la Loi cadre des Cultures ${ }^{41}$. Bref, la loi sur le cinéma n'est pas prioritaire alors que la production a explosé avec le numérique. La mobilisation de tous les acteurs du monde du cinéma bolivien a été vaine, à ce jour.

Il est indispensable de rappeler les conditions prohibitives des labels contrôlés par les multinationales qui dominent le circuit du numérique $(600$ \$ par séance indépendamment du nombre de spectateurs) témoins de l'obsolescence normative ${ }^{42}$ alors que la discursivité nationale dominante martèle l'opinion publique du "processus de décolonisation " politique, économique, social et sociétal. Mais, face à l'impossibilité d'accéder à des fonds publics nationaux, les cinéastes ont trouvé dans la créativité et le cinéma indépendants une alternative. Ils brillent par eux-mêmes mais leurs aventures sont en solitaires, abandonnés par l'État.

20 Affirmer que le numérique a démocratisé l'accès aux moyens de production cinématographique n'est pas une nouveauté dans les pays andins (ni ailleurs dans le monde). On observe une hausse significative des productions audiovisuelles aux formats et aux finalités multiples. Ce qui est nouveau, dans les Andes, c'est que l'on assiste à une sorte de décentralisation spontanée vers les régions, de la production cinématographique associée historiquement aux capitales, Bogotá, Lima, La Paz ou Quito. Les cinéastes des périphéries ont profité d'un format plus souple, moins cher et accessible à tous, professionnels ou non-professionnels. On constate aussi que la qualité technique, la rigueur que requièrent le langage de la création et la production cinématographiques, ne s'est pas améliorée avec l'arrivée du numérique. Bien au contraire.

21 La nouveauté réside dans la multiplication de nouveaux circuits de diffusion: organisations sociales, peuples autochtones, jeunes militants du milieu étudiant ou politique, travailleurs syndiqués, femmes, etc. Internet, YouTube, Facebook renforcent cette tendance. L'impact quantitatif et qualitatif s'observe dans la multiplication de rencontres et de festivals provinciaux, régionaux ou sous-régionaux (cinéma indigène, cinéma de genre, etc.). Mais, l'État, en Bolivie, en Colombie, en Équateur et au Pérou, non seulement a été incapable de (et rétif à) mettre en place une législation adaptée à la création, la production et la diffusion cinématographiques mais il s'avère autiste à la transformation de la société qui est pluriethnique et multiculturelle. Il est incapable, à ce jour, y compris en Bolivie qui se positionne en modèle de construction d'un État d'un nouveau type, de répondre à une des principales missions (pourtant inscrite dans les lois de cinéma de ces pays) : le développement de la culture et de l'identité collective, dont le cinéma est un des passeurs. 


\section{NOTES}

1. Dès le début des années 1990, reconnaissance de la plurinationalité et de la multiculturalité dans les pays andins, objets de notre étude. Instauration d'un État plurinational en Bolivie (Constitution de 2009).

2. Laberinto : une co-production des Nations Unies (PNUD), Coopération espagnole et Ambassade royale des Pays-Bas. Il s'agit de 7 DVD, correspondant aux 7 chapitres du Rapport des Nations Unies, Informe nacional sobre desarrollo humano, présentés sous forme de dialogue entre experts dans un but didactique. À titre d'exemples: Democracia y Gas, Tierra/Territorio; Autonomías/ discriminación ; Justicia. Símbolos y Héroes. Réalisateur : Juan Carlos Valdivia.

3. Rappelons toutefois le passage de nombreux cinéastes latino-américains au Chili (avant le coup d'état de Pinochet), en Europe ou à Cuba à l'Escuela Internacional de Cine y Televisión de San Antonio de los Baños.

4. Dans l'étude réalisée en 2005, Dinámica económica de la cultura en Bolivia, dont l'objet est d'évaluer l'impact économique des industries culturelles en Bolivie, les auteurs constatent que la télévision est positionnée loin devant la presse écrite d'une part, et que, d'autre part, elle a "réduit la fréquentation des salles de cinéma dans les zones urbaines, surtout dans celles qui ne peuvent organiser des premières » (cf. Eduardo López Z., Erick Torrico V., Alejandra Baldivia R., Dinámica económica de la cultura en Bolivia, Bogotá, Ed. Convenio Andrés Bello, 2005, p. 134 ; nous traduisons).

5. Les salles de cinéma en Bolivie sont passées de 120 à 30 entre 1986 et 1997. Source : SUZS Pedro, "Apuntes sobre cine boliviano" in Teresa Toledo (coord.), Made in Spanish 98: la distribución y la exhibición en Iberoamérica, San Sebastián, Festival internacional de cine de Donostia, 1998, cité dans : Eduardo López Z., Erick Torrico V., Alejandra Baldivia R., op. cit., p. 140. Dans les années 2000, ce sont les cine centers (multiplexes) qui n'ont fait qu'accélérer la fermeture des salles.

6. Par exemple, entre 2006 et 2016, l'Argentine a produit 564 films, le Brésil, 491 et le Mexique 345 (source : [http://www.programaibermedia.com].

7. GETINO Octavio, «Progrès des politiques d'intégration des cinématographies ibéroaméricaines ", Cinémas d'Amérique latine, Paris, 2011, n 19, p. 90-94.

8. [www.programaibermedia.com/el-marco-legal/legislacion-por-paises/].

9. [www.segib.org]. Préambule à l'Accord.

10. Ibid.

11. Il s'agit de l'Argentine, de Cuba, de l'Équateur, du Mexique, du Nicaragua, de Panama, du Pérou, du Venezuela, de la République dominicaine et du Brésil. Aujourd'hui, 20 pays ont signé l'Accord.

12. [www.segib.org]. Article 1 de l'Accord.

13. Protocolo de enmienda. Acuerdo latinoamericano de coproducción cinematográfica, (2000), (2006), Art. II. [www.boe.es]. núm. 143, de 16 de junio de 2017, pp. 49591-49595. Amendements des articles III et V.

14. Ibid.

15. [www.caaci.int]. Article premier de la Convention d'intégration cinématographique ibéroaméricaine.

16. Deux initiatives infra régionales sont à signaler: la Réunion spécialisée des autorités cinématographiques et audiovisuelles du Mercosur (RECAM) en 2003 et CINERGIA en 2004, un fonds de développement de l'audiovisuel pour l'Amérique centrale et les Caraïbes. 
17. [www.segib.org].

18. Notons que les PME du cinéma ibéro-américain regroupées au sein de la FIPCA (Fédération ibéro-américaine des producteurs cinématographiques et audiovisuels) participent activement à ce programme.

19. GETINO Octavio, op. cit., p. 93.

20. Colombie : Loi 814 du 2 juillet 2003. Soulignons la dotation d'un Fonds pour le développement cinématographique (art. 11 chap. II). Au moins $70 \%$ de ce fonds est destiné à la création, à la production, à la co-production et à la réalisation de longs métrages et de courts métrages colombiens.

21. Équateur : Loi 2006-29 du 3 février 2006. Ce sont des mesures comparables à celles prévues dans les lois péruvienne, colombienne et bolivienne: une politique volontariste d'accompagnement financier pour la création, la production et la diffusion d'oeuvres cinématographiques nationales sur des formats de tout type.

22. BEDOYA Ricardo, Historia de los medios de comunicación en el Perú : Siglo XXI. El cinema sonoro en el Perú, Lima, Fondo editorial, Universidad de Lima, 2013, p. 231.

23. Ajoutons à cela la récession économique des dernières années de la présidence d'Albert Fujimori et la fermeture des salles de cinéma et de spectacles dans les régions de l'intérieur du Pérou.

24. Anteproyecto de ley de la Cinematografía y el Audiovisual peruano. exposición de motivos, [http:// www.leyes.congreso.gob.pe].

25. Article 54 du décret législatif $n^{\circ} 776$, ibid.

26. Ibid.

27. Luis Espinal, Amalia Gallardo, Pero Zusz, Carlos Mesa, Alfonso Gumucio, Raquel Romero, entre autres.

28. Ley $\mathrm{N}^{\circ} 1302$, Chapitre II, art. 5 et 6. Gaceta Oficial de Bolivia, La Paz, 20/12/1991, [ www.presidencia.gob.bo].

29. Ibid.

30. Ibid.

31. GUERRA VELAZCO Gerardo (producteur et distributeur) in «El cine boliviano aún es emprendimiento personal ", La Razón, La Paz, 20/03/2016.

32. Association des travailleurs de l'image en Bolivie (ATIB), Association des cinéastes de Bolivie (ASOCINE), mouvement du nouveau cinéma bolivien (MNCVB), Association des cinéastes de Santa Cruz, Festival des cinémas des droits de l'homme à Sucre, Centre de formation et réalisation cinématographique (CEFREC), Coordination Audiovisuelle Indigène et Originaire de Bolivie (CAIB), etc.

33. Congrès du cinéma audiovisuel, avril 2013 à Cochabamba.

34. NCPE, adoptée par référendum le 9 février 2009, affirme que la Bolivie est un État plurinational démocratique (art. 1), que la souveraineté réside dans le peuple bolivien et s'exerce de façon directe et déléguée (art.7), qu'il existe plusieurs formes d'exercer la démocratie (art. 11) et que les Boliviens et les Boliviennes ont le droit à la formation, l'exercice et le contrôle du pouvoir politique (art. 26).

35. AGUILAR Gioconda (responsable de la proposition des associations de cinéma), La Revista, $\mathrm{n}$ - 31, 21/11/2017 [www.cinemascine.net].

36. Inaugurés dans les capitales régionales Cochabamba en 2009, à Santa Cruz, La Paz, etc., ces Cine centers ou Multicines proposent principalement du cinéma hollywoodien avec deux séances les mercredis pour le prix d'une.

37. ¿Quién mató a la llamita blanca? (Rodrigo Bellot, 2006), Zona Sur (Juan Carlos Valdivia, 2009), Hospital Obrero de (Germán Monje, 2009). 
38. Nous faisons référence au cinéma dit «populaire » et urbain, de productions digitales. On est passé de 4 à 5 films dans les années quatre-vingt et quatre-vingt-dix à une cinquantaine et plus dans les années 2000 .

39. No veo España (Ariel Coca, 2009), Gud Busines (Tonchy Antezana, 2010), par exemple.

40. Une journée du cinéma bolivien est organisée chaque année sur tout le territoire national pour alerter l'opinion publique sur l'urgence d'une nouvelle Loi.

41. Déclaration de Marko Machicado (ministre des cultures) in « ¿Ley del cine ahora? ", Ramona, Opinión, Cochabamba, 20/03/2016.

42. Un film national à grand succès aujourd'hui réalise au plus 15000 entrées, beaucoup moins que la vente généralisée des films « pirates».

\section{RÉSUMÉS}

Les changements sociétaux et politiques des années 2000 dans les pays andins coïncident avec le passage du celluloïd au numérique. La vidéo est devenue le principal media alternatif d'expression audiovisuelle. Elle démultiplie les créations dans tous les genres cinématographiques de plus ou moins bonne qualité. Demandant peu d'investissement, informel (pratiquement pas encore encadré par l'État), il est au cinéma ce que l'économie informelle est à l'économie formelle. Il est aussi le support avec lequel se sont formés de nombreux cinéastes reconnus. D'autres faits participent à la généralisation de la vidéo et du numérique : prolifération du DVD, des vidéo-clubs, des chaînes de télévisions privées, disparition progressive des salles commerciales et réduction des aides publiques (quand il y en avait). Existe-t-il au niveau continental une volonté des États de protéger la création cinématographique? Les législations évoluent-elles en Bolivie, en Colombie, en Équateur, au Pérou au rythme soutenu des productions numériques? Quelles mesures, si elles existent, sont prises institutionnellement pour développer, financer et protéger la création cinématographique? Tel est l'objet de notre réflexion.

Los cambios societales y políticos de los años 2000 en los países andinos coinciden con el paso del celuloide al soporte digital. Se transformó el video en el principal medio alternativo de expresión audiovisual. Se han multiplicado las creaciones en todos los géneros cinematográficos de mejor o peor calidad. Precisando de poca inversión, informal (todavía carente de un marco legal), es al cine lo que es la economía informal a la economía real. Es también el medio de formación de numerosos directores reconocidos. Participan a la generalización del video, la extensión del uso del DVD, los videoclubes, los canales privados de televisión, la desaparición progresiva de las salas comerciales y la reducción de las ayudas públicas (cuando había). ¿Existe la voluntad de los Estados, a nivel latinoamericano, de proteger la creación cinematográfica? ¿Van evolucionando las legislaciones en Bolivia, Colombia, Ecuador, Perú al ritmo sostenido de las producciones digitales? ¿Qué tipo de medidas se toman institucionalmente para fomentar, financiar y proteger la creación cinematográfica? Éste es el objeto de nuestro análisis. 
INDEX

Mots-clés : cinéma, numérique, pays andins, législation, création

Palabras claves : cine, digital, países andinos, legislación, creación

\section{AUTEUR}

CHRISTINE DELFOUR

Université Paris-Est Marne-la-Vallée, EMHIS-LISAA 